\title{
Formulation, Physical Characterization and Antioxidant Potential of Silymarin Suspensions and Emulsions
}

\author{
Arooj Amna ${ }^{a}$, Nazish Jahan ${ }^{a}$, Khalil-ur-Rehman ${ }^{b}$ and Zill-e-Huma ${ }^{c *}$ \\ ${ }^{a}$ Department of Chemistry, University of Agriculture, Faisalabad, Pakistan \\ ${ }^{b}$ Department of Biochemistry, Riphah International University, Faisalabad, Pakistan \\ ${ }^{c}$ Department of Chemistry, Riphah International University, Faisalabad, Pakistan
}

(received January 3, 2019; revised December 26, 2020; accepted January 6, 2021)

\begin{abstract}
Silymarin, is a therapeutically important flavonoid act as a hepatoprotective agent. However, has a positive effect on metabolism act as a hydrophobic drug but has a very low bioavailability. The aim of the present study was to prepare appropriate formulations of Silymarin in order to enhance its bioavailability. The natural suspending agents like (Xanthan gum, Tragacanth gum, Acacia gum and Iranian gum) were used for the formulation of suspensions, while emulsions were prepared with the combination of surfactant, co-surfactant and oil. The formulations were evaluated for their physical stability, $\mathrm{pH}$, refractive index and conductivity. Among different formulations and suspensions prepared with xanthan gum as areen stabilizing agents were most stable. Emulsions formulated with tween 80 as a surfactant, polyethylene glycol as co-surfactant and olive oil were clear and stable for more than six months. The refractive index, $\mathrm{pH}$ and conductivity of the most stable suspension and emulsions were $1.347,6.9$ and $0.18 \mathrm{~s} / \mathrm{m}$ and 1.43 , $6.9,0.01 \mathrm{~s} / \mathrm{m}$ respectively. It was concluded that xanthan gum and tween 80 with polyethylene glycol has a good potential to enhance the therapeutic efficiency and stability of silymarin suspension and emulsion.
\end{abstract}

Keywords: silymarin, low bioavailability, suspensions, emulsions, stability

\section{Introduction}

Silymarin (SM), an extract of Silybum marianum (L.), contains a mixture of four flavonolignan isomers: silibinin (70-80\%), silycristin (20\%), silydianin (10\%) and isosilybin (0.5\%) (Yang et al., 2015). Silymarin is found effective clinically to treat a variety of liver disorders, including acute and chronic viral hepatitis and due to phenolic structures have an antioxidant effect and inhibit free-radical mediated processes (Lahlah et al., 2012; Wu et al., 2006). However, the effectiveness of silymarin as a hepatoprotective drug is limited due to its poor water solubility and low bioavailability. For this purpose, oral formulations containing therapeutically active extract (silymarin) were prepared in the form of suspensions and emulsions with an objective to enhance its solubility and bioavailability. The natural gums were used for the formulation of suspensions and they can be an alternative to synthetic suspending agents. These formulations can be easily prepared, green and are costeffective. For the preparation of emulsions, several combinations of surfactants, co-surfactants and oil were used.

Herbal medicines have been widely utilized as effective remedies for the treatment, control and management of

*Author for correspondence; E-mail: zilehuma098@gmail.com a variety of ailments since prehistoric times (Chikezie et al., 2015; Jo et al., 2013). The World Health Organization (WHO) has reported that $80 \%$ of the developing world's population relies on traditional medicines for therapy (Mahomoodally, 2013). It has been estimated that approximately $60-70 \%$ of the drug molecules are insufficiently soluble in aqueous media and this is a limiting factor for in vivo bioavailability after oral administration (Jahan et al., 2016; Khadka et al., 2014). Therefore, to improve oral drug absorption and bioavailability of drugs a number of approaches have been taken up which include the use of absorption enhancers, prodrugs and permeability enhancing dosage forms such as liposomes and emulsions.

Suspensions deliver high drug concentration through a simple preparation procedure (Rajan et al., 2013). In the pharmaceutical suspension just like other disperse systems, the insoluble solid particles are dispersed in the liquid medium (Sudam et al., 2012). The xanthan gum, Acacia and cellulose derivatives are used as suspending agents and surfactants to synthesize a deflocculated system even when used in low concentrations (Barhate et al., 2014). Emulsions are used as a vehicle for the administration of drugs (Kumar and Bhopal, 2012). They improve the solubility, dissolution and oral absorption of poorly water-soluble 
drugs (Gundogdu et al., 2013). Emulsions can be used for targeted drug delivery and for efficient interaction with the receptor (Pavela et al., 2019). Three components are the basic requirement to form an emulsion, an oil phase, an aqueous phase and a surfactant (Sharma et al., 2016). The aim of this study was to enhance the bioavailability of silymarin by preparing the suspension and emulsions using different suspending agents, surfactants, co-surfactants and oils.

\section{Materials and Methods}

Preparation of plant extract. The silymarin was extracted from seeds of Silybum marianum commonly known as milk thistle by using the soxhlet technique. The seeds were defatted with n-hexane and extract was prepared with ethanol. The filtrate was evaporated in the rotary apparatus to get powdered silymarin and was stored in a refrigerator for further use.

Preparation of suspensions. The natural gums (xanthan, tragacanth, Acacia and Iranian) were used as green suspending agents as well as surfactants for the preparation of suspensions. Several combinations of natural gums, sodium lauryl sulphate (SLS) and ethyl alcohol with silymarin were formulated (Jaganath et al., 2011). Natural gums were dissolved in the distilled water, sodium lauryl sulphate, then silymarin, and ethanol were added into this mixture with continuous shaking. The composition of all the suspensions is given in Table 1.

Preparation of emulsions. The emulsions were formulated with tween 80 as a surfactant, monoethylene glycol (MEG), diethylene glycol (DEG), polyethylene glycol (PEG), ethanol as cosurfactants and olive oil as oil phase. A mixture of surfactant tween 80 was prepared with various cosurfactant, diethylene glycol (DEG), polyethylene glycol (PEG) etc. For the formulation of emulsions, silymarin was added into the surfactant, cosurfactant and water was added with continuous stirring (Osanloo et al., 2017). The composition of all the emulsions is given in Table 2.

Evaluation of physical parameters. Conductivity measurements. The electrical conductivity of suspensions and emulsions was measured with a conductivity meter.

Determination of $\mathbf{p H}$. The $\mathrm{pH}$ values for formulations were determined at $25^{\circ} \mathrm{C}$ by pH meter (HI2211 pH/ORP Meter). All measurements were carried out in triplicate.
Table 1. Composition of silymarin suspensions

\begin{tabular}{lccll}
\hline \hline Ingredients & S-1 & S-2 & S-3 & S-4 \\
\hline Xanthan (mg) & 50 & - & - & - \\
Tragacanth (mg) & - & 125 & - & - \\
Acacia $(\mathrm{mg})$ & - & - & 125 & - \\
Iranian (mg) & - & - & - & 125 \\
Water (mL) & 5 & 5 & 5 & 5 \\
Ethanol (mL) & 5 & 5 & 5 & 5 \\
SLS (mg) & 150 & 150 & 150 & 150 \\
Silymarin (mg) & 150 & 150 & 150 & 150 \\
\hline \hline
\end{tabular}

$\mathrm{SLS}=$ sodium lauryl sulphate

Table 2. Composition of silymarin emulsions

\begin{tabular}{llllll}
\hline \hline Ingredients & E-1 & E-2 & E-3 & E-4 & E5 \\
\hline Tween $80(\mathrm{~mL})$ & 5 & 10 & 5 & 5 & 5 \\
MEG $(\mathrm{mL})$ & 8 & - & - & - & - \\
DEG $(\mathrm{mL})$ & - & - & 8 & - & - \\
PEG $(\mathrm{mL})$ & - & - & - & - & 8 \\
Ethanol $(\mathrm{mL})$ & 5 & 5 & 5 & 5 & 5 \\
Oil $(\mathrm{mL})$ & 5 & 5 & 5 & 5 & 5 \\
Water $(\mathrm{mL})$ & 7 & 7 & 7 & 7 & 7 \\
Silymarin $(\mathrm{mg})$ & 150 & 150 & 150 & 150 & 150 \\
\hline \hline
\end{tabular}

MEG=monoethylene glycol; DEG=diethylene glycol; PEG=polyethylene glycol

Determination of refractive index. The refractive index of all formulations was measured with the help of a refractometer.

Antioxidant potential. The antioxidant potential was determined by the 2,2-diphenyl-1-picrylhydrazyl (DPPH) free radical quenching potential assay. Five different concentrations of emulsions, suspensions, plant extract and standard (ascorbic acid) in the range of 0.02 to 0.1 $\mathrm{mg} / \mathrm{mL}$ were prepared. Then $3 \mathrm{~mL}$ of these concentrations were taken and added to a freshly prepared solution of $0.1 \mathrm{mM} \mathrm{DPPH}$. The solutions were left for $30 \mathrm{~min}$ at room temperature and absorption was measured by using UV-Visible spectrophotometer (UV$1100)$ at $517 \mathrm{~nm}$. The percentage inhibition of DPPH radical was calculated by using the following formula:

$\%$ of $\mathrm{DPPH}=[1-\mathrm{A} 1 / \mathrm{A} 0] \times 100$

where:

$\mathrm{A} 1=$ Absorbance of sample; $\mathrm{A} 0=\mathrm{Absorbance}$ of control (Aslam et al., 2012)

\section{Results and Discussion}

Silymarin is utilized for the treatment of several liver diseases but this drug has poor aqueous solubility and 
therefore suffers from low bioavailability so, to overcome these shortcomings and it is incorporated into various formulations. After the preparation of emulsions, all the formulations were subjected to various evaluation tests such as conductivity, $\mathrm{pH}$, refractive index and physical stability. The visual inspection of all the formulations were carried out for a period of six months by drawing formulations sample at weekly interval for the first month and monthly interval for the subsequent months. The visual observation showed no evidence of phase separation. The physical stability was evaluated by visual inspection.

With the increase in demand for natural excipients, it is necessary to explore the newer sources of gums to meet the industrial demands (Sudam et al., 2012). During this research study, various suspensions were prepared by using natural gums such as Acacia, xanthan, tragacanth, as suspending agents (Eraga et al., 2014). The suspending properties of all the gums were also compared to get the best green suspending agent.

The suspension prepared with xanthan gum showed maximum stability followed by tragacanth gum, while suspensions formulated with gum Acacia and Iranian gum showed less stability. The viscosity of suspension containing a low concentration of the gum was low. The flow rates were inversely proportional to the viscosity of the suspension. The small amount $(25 \mathrm{mg})$ of xanthan gum was enough to formulate stable suspensions for the time of three months. When the amount of gum was increased up to $50 \mathrm{mg}$, the suspensions showed stability for more than six months. Xanthan gum is soluble in water and imparts high viscosity at low concentrations (Devrim et al., 2011). The tragacanth gum has also shown better results. The xanthan gum showed the best-suspending properties for the formulation of suspension with silymarin. The three dimensional network formed by the associated chains makes xanthan gum an efficient stabilizer for suspensions and emulsions. The polymer when coming in contact with water get hydrate and forms a gel and sustained the drug over a long period e.g. guar gums and xanthan gums. The suspensions prepared with Acacia and tragacanth gums were not stable for more than three months.

The formulations containing lower concentrations of gums were easily redispersed after shaking and no deposit was observed. Natural gums being hydrophilic colloids form a dispersion when coming in contact with water and increase the viscosity of the continuous phase.
The solid particles remain suspended in it for a long time. Gums can be used for sustain drug release. The changes in the $\mathrm{pH}$, conductivity, refractive index and colour of suspensions prepared with different concentrations of gums (xanthan, tragacantha, Acacia and Iranian), ethanol and sodium lauryl sulphate were recorded. The $\mathrm{pH}$ of all the suspensions was found between 6.8-7.4 Fig. 1. The $\mathrm{pH}$ of various formulations was found suitable for oral administration (Shakya et al., 2014). The conductivity of suspensions was varied between $0.18 \mathrm{~s} / \mathrm{m}$ and $0.30 \mathrm{~s} / \mathrm{m}$ Fig. 2. The refractive index was increased from 1.347 to 1.351 in $\mu$ Fig. 3 .

The bioavailability and permeability of poorly water soluble drugs has been enhanced through lipid-based formulations (Alam et al., 2015). Emulsions have the ability to enhance the solubility of drug molecules and

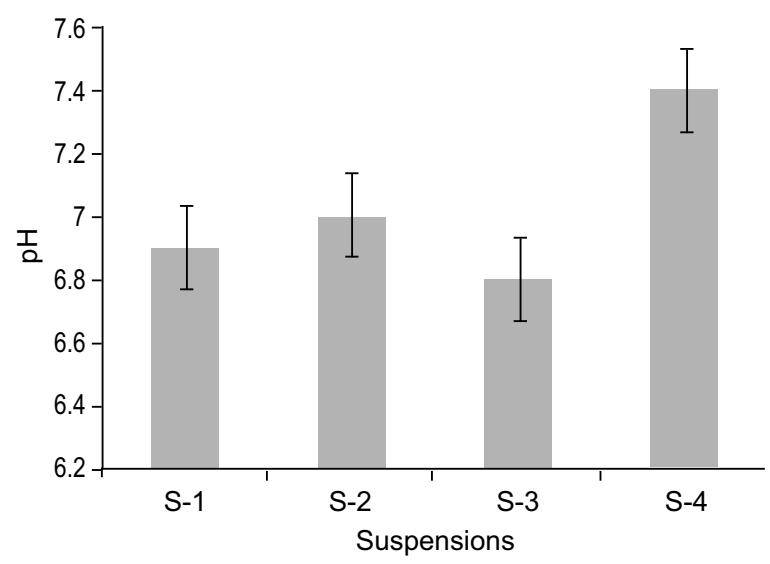

Fig. 1. $\mathrm{pH}$ of suspensions.

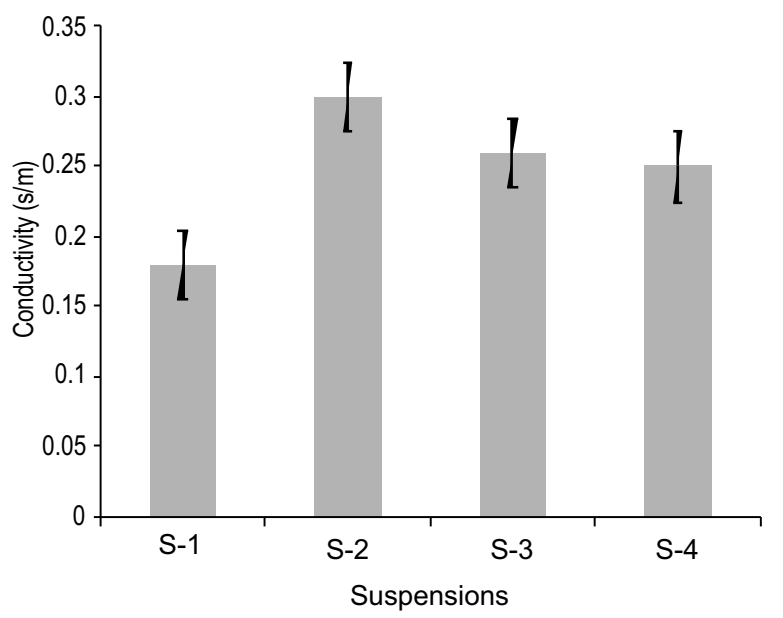

Fig. 2. Conductivity of suspensions. 


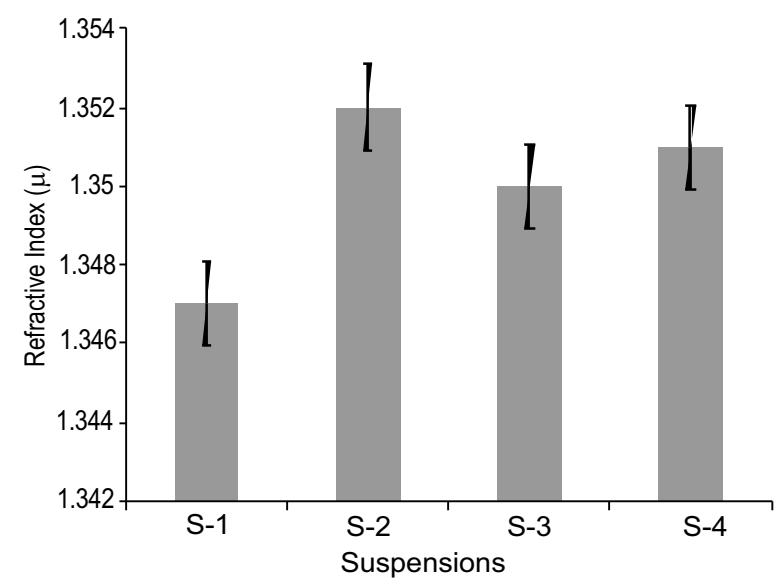

Fig. 3. Refractive index of suspensions.

are used extensively as drug carriers (Li et al., 2014). They are clear, stable and isotropic liquid mixtures of oil, surfactant and water, often in combination with a cosurfactant (Singh et al., 2011). Emulsifiers hinder the coalescence among approaching oil droplets by producing a repulsive electrical force. The emulsions were formulated by using tween 80 (surfactant), monoethylene glycol, diethylene glycol and polyethylene glycol (co-surfactant) as emulsifiers with olive oil. The emulsifying properties of all the emulsifiers were compared and the amount of drug $(150 \mathrm{mg})$ was constant and olive oil was used as the oil phase for all the emulsions. Oil presents a high amount of free fatty acids that can act as co-surfactants producing an oil phase with superior properties (Kelmann et al., 2007).

The emulsions formulated with tween 80 , polyethylene glycol and olive oil were stable for six months. The emulsions were inspected visually to observe any change or flocculation. For that purpose and all the formulations were inspected on regular basis for a period of six months by drawing emulsions sample at the daily intervals for the first month and at monthly intervals for the subsequent months. The emulsions prepared with polyethylene glycol did not show any flocculation or phase separation for six months. The emulsions formulated with monoethylene glycol as cosurfactant were stable for three months while that with diethylene glycol was stable for four months. The emulsions were also formulated without any surfactant and they were stable for one-two months only. The volume of tween 80 was also increased from $5 \mathrm{~mL}$ to $10 \mathrm{~mL}$ but no obvious effect was observed on the physical stability.
All the formulations have shown pale yellow colour and no change in the colour was observed for the whole period of study. The $\mathrm{pH}$ was ranged from 6.6 to 7.0, Fig. 4 (Moghimipour et al., 2013). The conductivity of the formulations (emulsions) ranged from 0.01 to 0.06 $\mathrm{s} / \mathrm{m}$ Fig. 5 . The refractive index was varied from 1.418 to 1.445 Fig. 6 . The refractive index (RI) of the formulations was found near to oil phase which indicates emulsions have water-in-oil structures (Gundogdu et al., 2013). The phase systems (o/w or w/o) of the emulsions were determined by measuring the conductivity of the emulsions. The conductivity of the emulsions showed the water-in-oil (w/o) structure of emulsions.

Antioxidants potential. Silymarin extract has an efficient antioxidant potential, therefore, provides protection against oxidative stress that causes several diseases such as aging, diabetes, cancer, cardiovascular

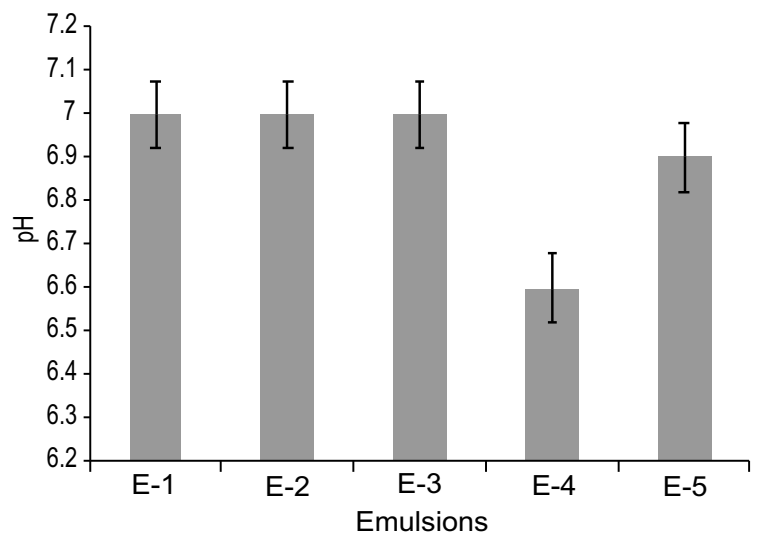

Fig. 4. pH of emulsions.

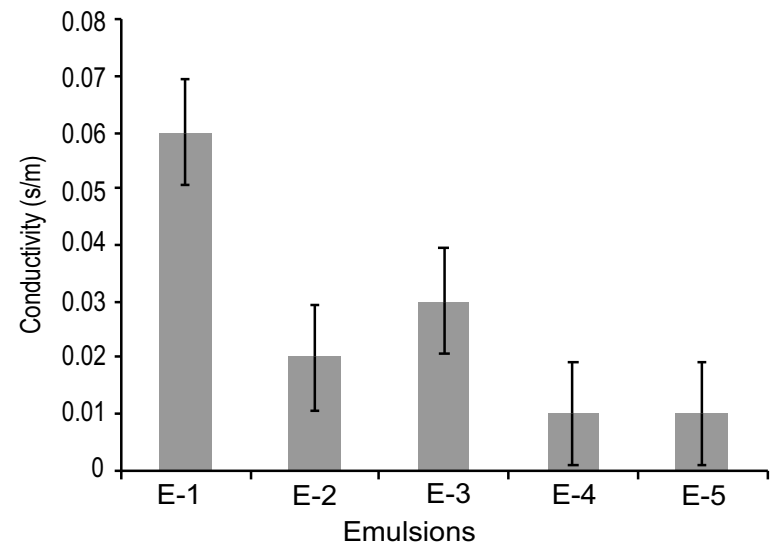

Fig. 5. Conductivity of emulsions. 


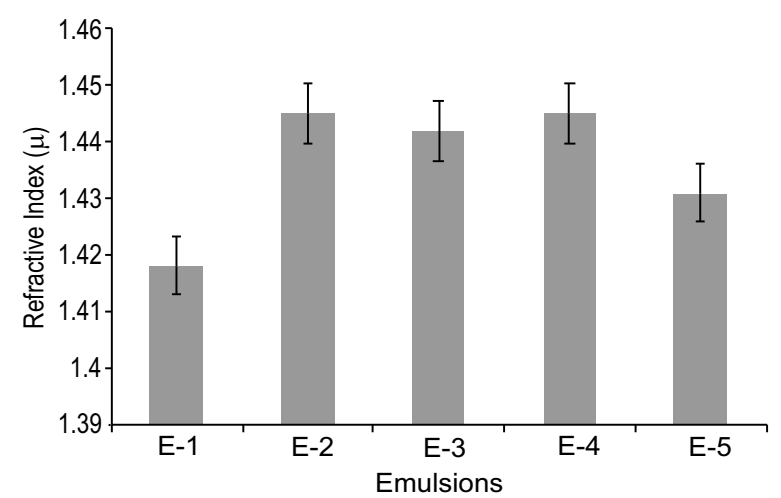

Fig. 6. Refractive index of emulsions.

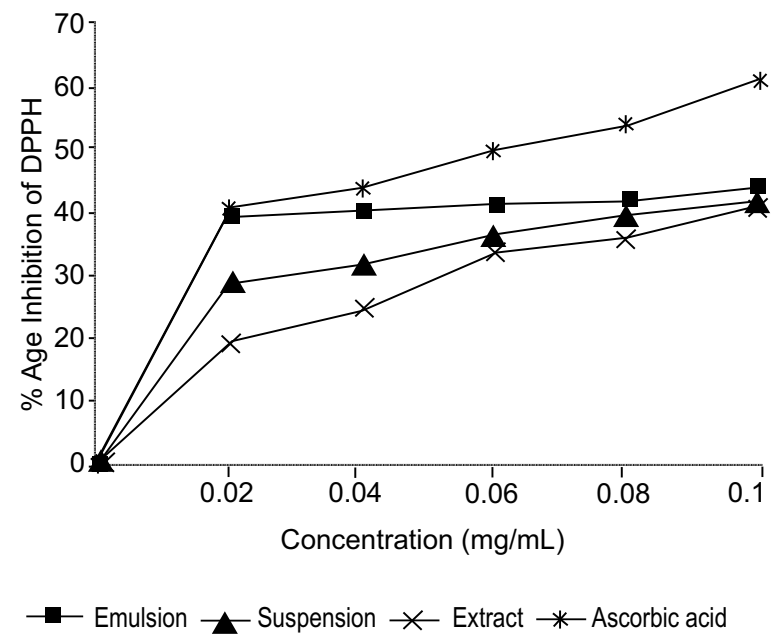

Fig. 7. Percentage inhibition of DPPH radical by emulsions and suspensions.

disorders and rheumatoid arthritis (Asghar and Masood, 2008; Kiruthiga et al., 2007). In order to evaluate the antioxidant potential of suspensions and emulsions, the DPPH radical scavenging assay was performed. DPPH scavenging activity is a well-known method to determine the antioxidant potential of medicinal plants in a short time period (Jahan et al., 2016).

The antioxidant potential was increased as the amount of formulations (suspensions and emulsions) was increased Fig. 7. The antioxidant potential of silymarin is due to higher levels of polyphenol compounds Asghar and Masood, (2008). The results revealed that the antioxidant potential of silymarin has been enhanced when it was incorporated into suspensions and emulsions as compared to the extract (Lin et al., 2021). With the formulation of suspensions and emulsions, in vitro antioxidant potential of plant extract has been enhanced. It may lead towards a better in vivo activity of plant extracts. The considerable inhibition of plant extracts is due to the presence of various antioxidants such as flavonols, xanthones, di-anthraquinones and flavans (Jahan et al., 2016). Phenolic compounds have quenching ability due to the presence of hydroxyl groups (Aslam et al., 2012). Among the formulations, the emulsions has higher potential as compared to suspensions. The silymarin showed high antioxidant capacity mainly due to its phenolic compounds and inhibits lipid peroxidation in plasma and RBC models. The silymarin can be used in preventing free radical-related diseases as a dietary natural antioxidant supplement (Asghar and Masood, 2008).

\section{Conclusion}

From all evaluated parameters it is concluded that xanthan gum has a promising potential as a suspending agent for the formulation of suspensions of silymarin. The emulsions prepared with tween 80 with polyethylene glycol as emulsifiers were most stable.

Conflict of Interest. The authors declare no conflict of interest.

\section{References}

Alam, M.S., Ali, M.S., Alam, M.I., Anwer, T., Safhi, M.M.A. 2015. Stability testing of beclomethasone dipropionate nanoemulsion. Tropical Journal of Pharmaceutical Research, 14: 15-20. https://www. tjpr.org/home/abstract.php?id=28\&aTitle=Stabili ty\%20testing\%20of\%20beclomethasone\%20dipr opionate \%20nanoemulsion.

Asghar, Z., Masood, Z. 2008. Evaluation of antioxidant properties of silymarin and its potential to inhibit peroxyl radicals in vitro. Pakistan Journal of Pharmaceutical Sciences, 21: 249-54. https:// pubmed.ncbi.nlm.nih.gov/18614420/.

Aslam, S., Jahan, N., Ali, S., Rahman, K.U. 2012. An innovative microwave-assisted extraction and antioxidant potential of polyphenols from different parts of Ocimum basilicum. Journal of Medicinal Plants Research, 6: 2150-59. https://doi.org/10. 5897/JMPR11.1444.

Barhate, A.N., Virkar, A.R., Beldar, G.B., Shinde, S. 2014. Evaluation of Delonix regia seed gum as suspending agent in paracetamol suspensions. International Journal of Pharmtech Research, 6: 530-36. http://sphinxsai.com/2014/PTVOL6/PT=18 
(530-536)AJ14.pdf.

Chikezie, P.C., Ojiako, O.A. 2015. Herbal medicine: yesterday, today and tomorrow. Alternative \& Integrative Medicine, 4: 2-5. https://www.hilaris publisher.com/open-access/herbal-medicineyesterday-today-and-tomorrow-2327-51621000195.pdf.

Devrim, B., Bozkir A., Canefe, K. 2011. Formulation and evaluation of re-constitutable suspensions containing ibuprofen-loaded eudragit microspheres. Acta Poloniae Pharmaceutica, 68: 593-99. https:// pubmed.ncbi.nlm.nih.gov/21796942/.

Eraga, S.O., Iwuagwu, M.A., Adikwu, M.U. 2014. Evaluation of the suspending properties of the coprecipitate of Irvingia gabonensis gum and gelatin. Tropical Journal of Pharmaceutical Research, 13: 843-48. https://www.researchgate. net/deref/http\%3A\%2F\%2Fdx.doi.org\%2F10.43 14\%2Ftjpr.v13i6.3.

Gundogdu, E., Baspinar, Y., Koksal, C., Ince, I., Karasulu, E. 2013. A micro-emulsion for the oral drug delivery of Pitavastatin evren. Pharmaceutica Analytica Acta, 4: 1-5. 10.4172/2153-2435.1000209.

Jaganath, S., Palanichamy, S., Buharym, S.H.M., Rajesh, M., Prabhu, C., Thirupathi, A.T. 2011. Preparation and evaluation of silymarin controlled release tablets prepared using natural gums. International Journal of Pharmaceutical Sciences and Nanotechnology, 4: 1368-72.

Jahan, N., Aslam, S., Rahman, K.U., Fazal, T., Anwar, F., Saher, R. 2016. Formulation and characterisation of nano-suspension of herbal extracts for enhanced antiradical potential. Journal of Experimental Nanoscience, 11: 72-80.

Kelmann, R.G., Kuminek, G., Teixeira, H.F., Koester, L.S. 2007. Carbamazepine parenteral nanoemulsions prepared by spontaneous emulsification process. International Journal of Pharmaceutics, 342: 231-9. https://doi.org/10.1016/j.ijpharm.2007. 05.004 .

Khadka, P., Ro, J., Kim, H., Kim, I., Kim, J.T., Kim, H., Cho, J.M., Yun, G., Lee, J. 2014. Pharmaceutical particle technologies: an approach to improve drug solubility, dissolution and bioavailability. Asian Journal of Pharmaceutical Sciences, 9: 304-16. https://doi.org/10.1016/j.ajps.2014.05.005.

Kiruthiga, P.V., Shafreen, R.B., Pandian, S.K., Arun, S., Govindu, S., Devi, K.P. 2007. Protective effect of silymarin on erythrocyte haemolysate against benzo(a) pyrene and exogenous reactive oxygen species $\left(\mathrm{H}_{2} \mathrm{O}_{2}\right)$ induced oxidative stress. Chemosphere, 68: 1511-18. https://doi.org/10.1016/ j.chemosphere.2007.03.015.

Kumar, P.V., Bhopal, A.K.P. 2012. Formulation design and evaluation of rutin loaded self-emulsifying drug delivery system (sedds) using edible oil. Asian Journal of Pharmaceutical and Clinical Research, 5: 76-78. https://www.researchgate.net/publication/ 231817103.

Lahlah, Z.F., Meziani, M., Maz, A. 2012. Silymarin natural antimicrobiol agent extracted from Silybum marianum. Journal of Academica, 2: 164-69.

Li, Y., Song, J., Tian, N., Cai, J., Huang, M., Xing, Q., Wang, Y., Wu, C., Hu, H. 2014. Improving oral bioavailability of metformin hydrochloride using water in oil micro-emulsions and analysis of phase behaviour after dilution. International Journal of Pharmaceutics, 473: 316-25. https://doi.org/10. 1016/j.ijpharm.2014.07.011.

Lin, D., Alan, L.K., Song, M., 2021. The role of mixing sequence in structuring $\mathrm{O} / \mathrm{W}$ emulsions and emulsion gels produced by electrostatic proteinpolysaccharide interactions between soy protein isolate-coated droplets and alginate molecules. Food Hydrocolloids, 113: 106537. https://www. sciencedirect.com/science/article/abs/pii/S02680 05X20329118.

Mahomoodally, M.F. 2013. Traditional medicines in Africa: an appraisal of ten potent African medicinal plants. Evidence-Based Complementary and Alternative Medicines, 2013:1-14. https://doi. org/10.1155/2013/617459.

Moghimipour, E., Salimi, A., Eftekhari, S. 2013. Design and characterization of micro-emulsion systems for naproxen. Advanced Pharmaceutical Bulletin, 3: 63-71. https://doi.org/10.5681/apb.2013.011.

Osanloo, M., Amir, A., Hassan, S., Mohammad, R.A., Fariba, E., Mohammad, M.S. 2017. Preparation and optimization nano-emulsion of Tarragon (Artemisia dracunculus) essential oil as effective herbal larvicide against Anopheles stephensi. Industrial Crops and Products, 109: 214-219. sciencedirect.com/science/article/abs/pii/S09266 69017305484.

Pavela, R., Benelli, G., Pavoni, L., Bonacucina, G., Cespi, M., Kevin, C., Iman, B., Mohammad, R.M., Giulio, L., Donato, R., Angelo, C., Filippo, M. 2019. Micro-emulsions for delivery of Apiaceae essential oils-Towards highly effective and ecofriendly mosquito larvicides? Industrial Crops and 
Products, 129: 631-640. https://www.science direct.com/science/article/abs/pii/S092666901 8310483.

Rajan, S.T., Sarathchandiran, I., Kadalmani, B. 2013. Evaluation of newly developed herbal oral suspension for spermatotoxic activity on male wistar albino rats. Journal of Pharmacy Research, 7: 347-351.

Rivera, J.O., Loya, A.M., Ceballos, R. 2013. Use of herbal medicines and implications for conventional drug therapy medical sciences. Alternative \& Integrative Medicines, 2: 1-6. https://www.hilaris publisher.com/open-access/use-of-herbalmedicines-and-implications-for-conventional-drugtherapy-medical-sciences-2327-5162.1000130.pdf.

Sharma, V.K., Koka, A., Yadav, J., Sharma, A.K., Keservani, R.K. 2016. Self-micro emulsifying drug delivery systems: a strategy to improve oral bioavailability. Ars Pharmaceutica, 57: 97-109. http://dx.doi. org/10.4321/S2340-989420160003 00001.

Singh, V., Bushettii, S.S., Raju, A.S., Ahmad, R., Singh, M., Bisht, A. 2011. Micro-emulsions as promising delivery systems: a review. Indian Journal of
Pharmaceutical Education and Research, 45: 392401. http://www.ijper.org/article/360.

Sudam, N., Manish, B., Ritesh, M., Sachin, P., Ratnaparkhi, M.P., Shilpa, C. 2012. Evaluation of various natural suspending agents for its suspending behaviour using paracetamol as model drug for suspension. Asian Journal of Pharmaceutical and Clinical Research, 5: 183-86. https://www.semantic scholar.org/paper/EVALUATION-OF-VARIOUSNATURAL-SUSPENDING-AGENTS-FORSudam-Manish/b 98 f 241758 e 883 f 50 e 09b72349d4f3b32324e8fa .

Wu, W., Wang, Y., Que, L. 2006. Enhanced bioavail ability of silymarin by self-micro-emulsifying drug delivery system. European Journal of Pharma ceutics and Biopharmaceutics, 63: 288-294. https:// doi.org/10.1016/j.ejpb.2005.12.005.

Yang, G., Zhao, Y., Feng, N., Zhang, Y., Liu, Y., Dang, B. 2015. Improved dissolution and bioavailability of silymarin delivered by a solid dispersion prepared using supercritical fluids. Asian Journal of Pharmaceutical Sciences, 10: 194-202. https:// doi.org/10.1016/j.ajps.2014.12.001. 